\title{
On Intelligent Extraction of an Internal Signal in a Wiener System Consisting of a Linear Block Followed by Hard-Nonlinearity
}

\author{
Kazys KAZLAUSKAS, Rimantas PUPEIKIS \\ Process Recognition Department, Institute of Mathematics and Informatics of Vilnius University \\ Akademijos 4, LT-08663 Vilnius, Lithuania \\ e-mail:kazys.kazlauskas@mii.vu.lt,rimantas.pupeikis@mii.vu.lt
}

Received: October 2011; accepted: August 2012

\begin{abstract}
The aim of the given paper is development of an approach based on reordering of observations to be processed for the extraction of an unmeasurable internal intermediate signal, that acts between linear dynamical and static nonlinear blocks of the Wiener system with hard-nonlinearity of the known structure. The technique based on the ordinary least squares (LS) and on data partition is used for the internal signal extraction. The results of numerical simulation and identification of a discrete-time Wiener system with five types of hard-nonlinearities, such as saturation, dead-zone, preload, backlash, and, discontinuous nonlinearity are given by computer.
\end{abstract}

Keywords: Wiener systems, hard-nonlinearity, internal signal, parametric identification, observations.

\section{Introduction}

In designing control systems, one ought to determine the type of uncertainties appearing in the dynamical system to be controlled. There are many types of uncertainties in control system description models. One of the main ones of them is the uncertainty arising due to nonlinearities of measurement devices and/or control actuators that significantly limit the performance of control systems (Åström and Wittenmark, 1996; Ljung, 1999). It is known (Selmic et al., 2003), that inaccuracy of mechanical components and nature of physical laws make actuators nonlinear devices. Usually, such nonlinearities can occur on the output of the system to be controlled inducing negative effects that might sometimes lead to unstable control (Schoukens et al., 2005). In such a case, Wiener models consisting of a linear dynamical system followed by a static nonlinear block are considered to be suitable for a broad spectrum of nonlinear processes (Wigren, 1993; Hagenblad, 1998; Ljung, 1999; Vörös, 2001; Pupeikis et al., 2003; Pupeikis, 2005a, 2005b, 2011). Nonlinearities, such as, friction, deadzone, saturation, preload, backlash and hysteresis are called hard-nonlinearities, and are common in most control systems, especially in electro-mechanical ones (Selmic et al., 2003). As noted in Hagenblad (1998), Vörös $(2001,2002,2010 a, 2010 b)$ in practice there exist the nonlinear dynamic systems 
with discontinuous asymmetric piecewise-linear characteristics having preloads and dead zones, too. It is known (Selmic et al., 2003) that most of these nonlinearities cannot be canceled or compensated using feedback linearization techniques because they appear in the feedforward path. On the other hand, it is known also, that most of them cannot be approximated by a low-order polynomial, and they are not readily invertible, too. Therefore, hard-nonlinearities have been a challenge for control system engineers for years (Åström and Wittenmark, 1996; Ljung, 1999; Selmic et al., 2003). Recently, static and dynamic compensators have been made up, and intelligent control techniques have been proposed for compensation of those nonlinearities: friction models and respective compensation techniques are given in Armstrong-Hélouvry et al. (1994), Canudas de Wit et al. (1995); a deadzone compensation approach in motion control systems has been established in Selmic and Lewis (2000); the backlash compensation technique has been found using dynamic inversion in Desoer and Shahruz (1986), Selmic and Lewis (2001), Selmic et al. (2003), Vörös (2011); the general actuator saturation compensator scheme has been developed in Åström and Wittenmark (1996), Selmic et al. (2003), Widanage et al. (2004), etc.

In general, various compensators have been tried to adjust the performance of electromechanical control systems by reducing parasitic effects of hard-nonlinearities. On the other hand, we describe here the common approach (Pupeikis et al., 2003) based on extraction of the unmeasurable internal intermediate signal, acting between linear and nonlinear blocks of the Wiener system (Hagenblad, 1998; Ljung, 1999; Vörös, 2010b) without designing special and complex enough compensators. To this end, we propose here to extract an unmeasurable internal signal that will be without parasitic effects. Afterwards, instead of measurable noisy output of the Wiener system affected by the respective hardnonlinearity, the extracted signal could be used for ordinary control of a linear dynamical system, free of the abovementioned unacceptable effects (Pupeikis et al., 2003).

In Section 2, a statement of the problem is presented. In Section 3, the common method is given for determining an auxiliary signal that corresponds to the extracted version of the internal one, that acts between both blocks of the Wiener system with respective hard-nonlinearity of known structure. In Section 4, an iterative procedure for extraction of an internal signal in a noisy environment is described. Section 5 presents the simulation and parametric identification results for different types of hard-nonlinearities. Section 6 contains conclusions.

\section{Statement of the Problem}

A control system to be considered consists of a linear block $G\left(q^{-1}, \boldsymbol{\Theta}\right)$ followed by a static nonlinearity $f(\cdot, \eta)$ (Fig. 1). The linear block is dynamic, time invariant, causal, and stable. It can be represented by a linear time-invariant dynamic system (LTI) with the function $G\left(q^{-1}, \boldsymbol{\Theta}\right)$ of known order as a rational function of the form

$$
G\left(q^{-1}, \boldsymbol{\Theta}\right)=\frac{b_{1} q^{-1}+\cdots+b_{m} q^{-m}}{1+a_{1} q^{-1}+\cdots+a_{m} q^{-m}}=\frac{B\left(q^{-1}, \mathbf{b}\right)}{1+A\left(q^{-1}, \mathbf{a}\right)}
$$




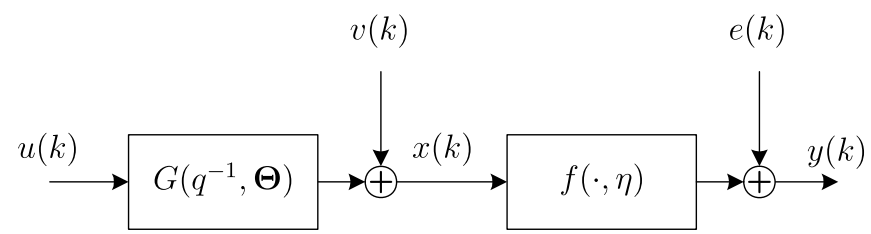

Fig. 1. The Wiener system with the process noise $v(k)$ and measurement noise $e(k)$. Signals: $u(k)$ is input, $y(k)$ is output, $x(k) \forall k=\overline{1, N}$ is an unmeasurable internal intermediate signal to be extracted.

with the finite number of parameters

$$
\begin{aligned}
& \boldsymbol{\Theta}^{T}=\left(b_{1}, \ldots, b_{m}, a_{1}, \ldots, a_{m}\right), \quad \mathbf{b}^{T}=\left(b_{1}, \ldots, b_{m}\right), \\
& \mathbf{a}^{T}=\left(a_{1}, \ldots, a_{m}\right),
\end{aligned}
$$

that are determined from the set $\boldsymbol{\Omega}$ of permissible parameter values $\boldsymbol{\Theta}$. Here $q^{-1}$ is the backward shift operator.

The set $\Omega$ is restricted by conditions on the stability of the respective difference equation with the finite number of parameters $\boldsymbol{\Theta}^{T}=\left(b_{1}, \ldots, b_{m}, a_{1}, \ldots, a_{m}\right)$ that are determined from the area of permissible parameter values. The unmeasurable intermediate signal

$$
x(k)=\frac{B\left(q^{-1}, \mathbf{b}\right)}{1+A\left(q^{-1}, \mathbf{a}\right)} u(k)+v(k),
$$

$\forall k=\overline{1, N}$ generated by (1) as a response to the input $u(k) \forall k=\overline{1, N}$, and corrupted by an additive noise $v(k) \forall k=\overline{1, N}$, is acting on a static nonlinear block $f(\cdot, \eta)$ with the vector of parameters $\eta$, i.e.,

$$
y(k)=f(x(k), \eta)+e(k),
$$

$\forall k=\overline{1, N}$. A nonlinear block represents a hard-nonlinearity of the known structure with unknown $\eta$, i.e., the saturation or dead-zone (Figs. 2a, 2b), preload or backlash (Figs. 2c, 2d) or any other nonlinearity even a discontinuous one (Fig. 3), that, according to formula (4), generates $y(k) \forall k=\overline{1, N}$ cutting off or changing some observations of the unmeasurable internal signal $x(k) \forall k=\overline{1, N}$ in a special way.

The process noise $v(k) \equiv \xi(k) \forall k=\overline{1, N}$ and the measurement noise $e(k) \equiv$ $\zeta(k) \forall k=\overline{1, N}$ are added to an internal intermediate signal $x(k) \forall k=\overline{1, N}$ and an output $y(k) \forall k=\overline{1, N}$, respectively; $\xi(k), \zeta(k)$ are mutually uncorrelated sequences of independent Gaussian variables with $E\{\xi(k)\}=0, E\{\zeta(k)\}=0, E\{\xi(k) \xi(k+\tau)\}=$ $\sigma_{\xi}^{2} \delta(\tau), E\{\zeta(k) \zeta(k+\tau)\}=\sigma_{\zeta}^{2} \delta(\tau) ; E\{\cdot\}$ is a mean value, $\sigma_{\zeta}^{2}, \sigma_{\xi}^{2}$ are variances of $\zeta$ and $\xi$, respectively, $\delta(\tau)$ is the Kronecker delta function.

The aim of the given paper is to avoid nonlinear distortions that appear in $y(k) \forall k=$ $\overline{1, N}$ by extracting the unmeasurable internal signal $x(k) \forall k=\overline{1, N}$, free from parasitic effects, instead of designing of special and complex enough compensators. 

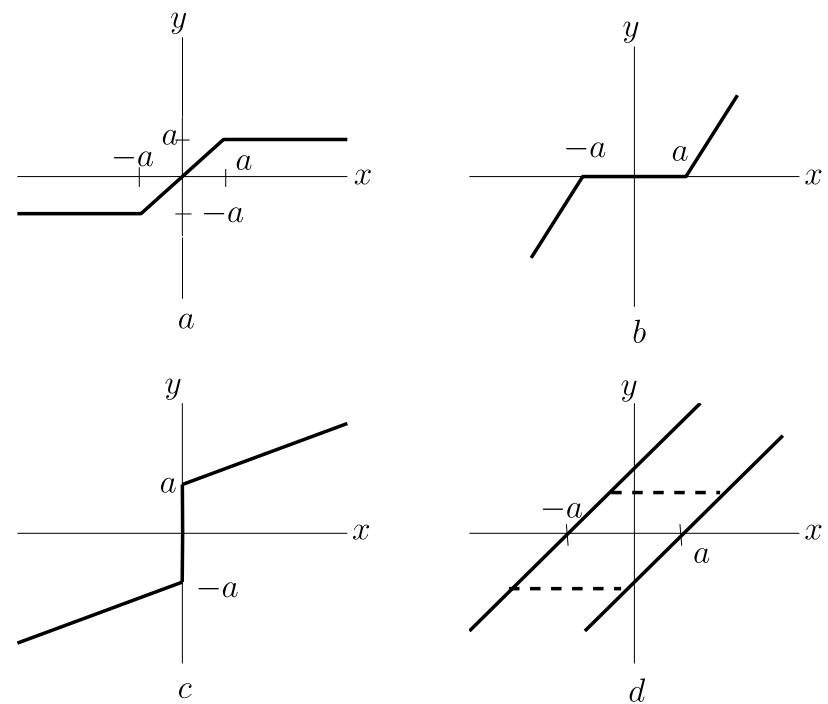

Fig. 2. Some examples of hard-nonlinearities: saturation (a), dead-zone (b), preload (c), backlash (d), $\eta \equiv a, a$ is a threshold.
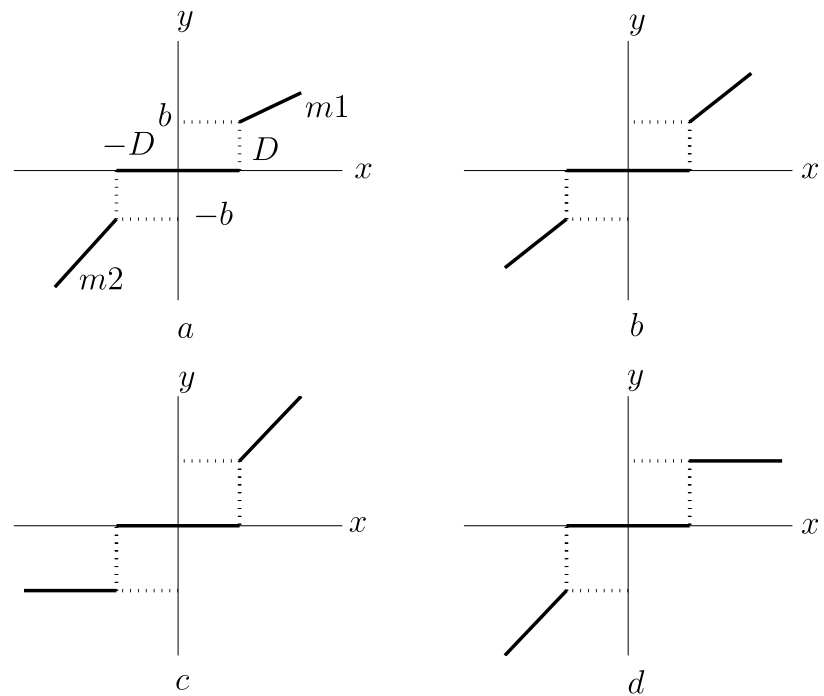

Fig. 3. Examples of discontinuous nonlinearities with the different leaning over linear segments (Vörös, 2001): $m_{1} \neq m_{2}(\mathrm{a}), m_{1}=m_{2}$ (b), $m_{2}=0$ (c), $m_{1}=0$ (d), $\eta=\left(m_{1}, m_{2}, b, D\right)^{T}$.

\section{Data Rearrangement}

Let us assume for simplicity that the process noise $v(k) \forall k=\overline{1, N}$ and the measurement noise $e(k) \forall k=\overline{1, N}$ are absent. To calculate an auxiliary signal $\hat{x}(k)$ (the estimate of unmeasurable $x(k)) \forall k \in \overline{1, N}$, one could approximate the model of the linear dynamic 
part of the Wiener system (1)-(4) by the finite impulse response (FIR) model of the form

$$
y(k)=\beta_{0}+\beta_{1} u(k)+\beta_{2} u(k-1)+\cdots+\beta_{\nu} u(k-\nu+1),
$$

$\forall k \in \overline{\nu, N}$, or using the expression in a matrix form

$$
\begin{aligned}
& \mathbf{Y}=\boldsymbol{\Lambda} \beta \\
& \mathbf{Y}=(y(\nu), y(\nu+1), \ldots, y(N-1), y(N))^{T}
\end{aligned}
$$

is the $(N-\nu+1) \times 1$ vector consisting of values $y(k)$,

$$
\boldsymbol{\Lambda}=\left[\begin{array}{ccccc}
1 & u(\nu) & \ldots & u(2) & u(1) \\
1 & u(\nu+1) & \ldots & u(3) & u(2) \\
\vdots & \vdots & \vdots & \vdots & \vdots \\
1 & u(N) & \ldots & u(N-\nu+2) & u(N-\nu+1)
\end{array}\right]
$$

is the full rank $(N-\nu+1) \times(\nu+1)$ regression matrix, consisting only of observations of the non-noisy input $u(k)$ (see Fig. 1);

$$
\beta^{T}=\left(\beta_{0}, \beta_{1} \ldots, \beta_{\nu}\right)
$$

is a $(\nu+1) \times 1$ vector of unknown parameters, $\nu$ is the order of the FIR filter that can be arbitrarily large but fixed.

The reason for the use of the FIR model is as follows: the dependence of some regressors on the process output will be facilitated, and the assumption of the ordinary LS that the regressors depend only on the non-noisy input signal, will be satisfied. This is the main consequence of replacing the initial transfer function $G\left(q^{-1}, \boldsymbol{\Theta}\right)$ of the linear part of the system by the FIR filter (5).

Next, let us rearrange the data in the vector $\mathbf{Y}$ in an ascending order of their values, reordering the associated rows of matrix $\Lambda$, too. One could carry out that by interchanging equations in the initial system (6). Then the vector $\mathbf{Y}$ and the matrix $\boldsymbol{\Lambda}$ should be partitioned into three data sets (Pupeikis, 2003): the left-hand data set

$$
\mathbf{Y}_{1}=\Lambda_{1} \beta
$$

the middle data set

$$
\mathbf{Y}_{2}=\Lambda_{2} \beta,
$$

and the right-hand data set according to the three regimes of respective nonlinearities

$$
\mathbf{Y}_{3}=\Lambda_{3} \beta
$$


Here $\mathbf{Y}_{1}, \mathbf{Y}_{2}, \mathbf{Y}_{3}$ are $N_{1} \times 1, N_{2} \times 1$ and $N_{3} \times 1$ vectors, respectively, $\boldsymbol{\Lambda}_{1}, \boldsymbol{\Lambda}_{2}, \boldsymbol{\Lambda}_{3}$ are $\left(N_{1}-\nu+1\right) \times(\nu+1), N_{2} \times(\nu+1)$ and $N_{3} \times(\nu+1)$ matrices, correspondingly, $N=N_{1}+N_{2}+N_{3}$.

Thus the initial system (6) is reordered into the system

$$
\tilde{\mathbf{Y}}=\tilde{\Lambda} \beta
$$

with

$$
\tilde{\mathbf{Y}}=\left[\begin{array}{l}
\mathbf{Y}_{1} \\
\mathbf{Y}_{2} \\
\mathbf{Y}_{3}
\end{array}\right],
$$

and

$$
\tilde{\boldsymbol{\Lambda}}=\left[\begin{array}{l}
\boldsymbol{\Lambda}_{1} \\
\boldsymbol{\Lambda}_{2} \\
\boldsymbol{\Lambda}_{3}
\end{array}\right],
$$

by simply interchanging equations in the initial system (6).

For the Wiener system with the saturation, the left-hand side data set $\mathbf{Y}_{1}\left(N_{1}\right.$ samples) consists of the reordered cut-off values $\tilde{y}(\tilde{k}) \forall k \in \overline{1, N_{1}}$, equal to negative $a$, the middle data set $\mathbf{Y}_{2}\left(N_{2}\right.$ samples $)$ of the reordered true values $\tilde{y}(\tilde{k}) \forall k \in \overline{1, N_{2}}$ higher than negative $a$, but lower than $a$, and the right-hand side data set $\mathbf{Y}_{3}\left(N_{3}\right.$ samples) consists of the reordered cut-off values $\tilde{y}(\tilde{k}) \forall k \in \overline{1, N_{3}}$ equal to $a$, if the measurement noise $e(k) \forall k=\overline{1, N}$ is absent. Here $0<a<\infty$ is the unknown threshold of saturation (Fig. 2a); $\tilde{k}$ is any integer $k$ rearranged in an ascending order, depending on the reordered values of observations $y(k) \forall k \in \overline{1, N}$, e.g., $\tilde{k}=1$ while $k=10$. Hence, the reordered cut-off observations with the same highest and positive value $a$ will be concentrated on the right-hand side set, while the reordered cut-off observations with the same lowest and negative value $-a$ on the left-hand side set. The observations of the middle data set of $\tilde{y}(\tilde{k})$ are coincident with the respective observations of the unmeasurable intermediate signal $x(k)$, i.e., $\tilde{y}(\tilde{k}) \equiv x(k)$ for $|x(k)|<a \forall k=\overline{1, N_{2}}$ because, in the case of saturation, the output signal $y(k) \forall k \in \overline{1, N}$ has been generated by the formula (Bai, 2002)

$$
y(k)=\frac{1+\operatorname{sign}(a-|x(k)|)}{2} x(k)+\frac{1+\operatorname{sign}(|x(k)|-a)}{2} a \operatorname{sign} x(k),
$$

$\forall k=\overline{1, N}$. Here sign is the standard sign function.

Note that, output signals $y(k) \forall k=\overline{1, N}$ of the dead-zone, preload, and backlash shown in Fig. 2 are generated by formula (Bai, 2002)

$$
y(k)=x(k)-a \operatorname{sign}(x(k))-\frac{1+\operatorname{sgn}(a-|x(k)|)}{2}(x(k)-a \operatorname{sign}(x(k)),
$$




$$
\begin{aligned}
& y(k)=x(k)+a \operatorname{sign}(x(k)), \\
& y(k)= \begin{cases}x(k)-a, & \text { if } x(k)-x(k-1)>0, \\
x(k)+a, & \text { if } x(k)-x(k-1)<0, \\
y(k-1), & \text { if } x(k)=x(k-1),\end{cases}
\end{aligned}
$$

respectively. The output of the discontinuous nonlinearity $y(k) \forall k=\overline{1, N}$ is calculated according to Vörös (2001)

$$
\begin{aligned}
& y(k)= \begin{cases}\tilde{y}(k), & \text { if }|x(k)|>D, \\
0, & \text { if }|x(k)|<D,\end{cases} \\
& \tilde{y}(k)=K(k)\{x(k)-D \operatorname{sign}[x(k)]\}+b \operatorname{sign}[x(k)],
\end{aligned}
$$

and

$$
K(k)=m_{1}+\left(m_{2}-m_{1}\right) h(k) .
$$

Here $\left|m_{1}\right|<\infty,\left|m_{2}\right|<\infty$ are respective coefficients that determine the leaning over the corresponding linear segments and $|b|<\infty$ is the preload constants (see Fig. 3); the switching function $h(k)$ has the form

$$
h(k) \equiv h[x(k)]= \begin{cases}0, & \text { if } x(k)>0 \\ 1, & \text { if } x(k)<0\end{cases}
$$

It follows that for the saturation (16), dead-zone (17), and discontinuous nonlinearity (20)-(23) there exist three regimes, in total. For the saturation (Fig. 2a), only the system of linear equations (11), based on true reordered values of $\tilde{x}(\tilde{k}) \equiv x(k) \forall k=\overline{1, N_{2}}$, is valid while systems (10) and (12) are not usable because of the presence of cutoff observations of $y(k)$. For the dead-zone (Fig. 2b) and the discontinuous nonlinearity (Fig. 3), the reordered observations of the left-hand side and right-hand side sets $\mathbf{Y}_{1}, \mathbf{Y}_{3}$ are not cut-off by the corresponding nonlinearity, while the observations of the middle data set $\mathbf{Y}_{2}$ of $\tilde{y}(k)$ are cut-off and equal to zero, i.e., $y(k) \equiv 0$ for $|x(k)|<a$ or for $|x(k)|<D$, respectively. Here $0<a<\infty, 0<D<\infty$ are dead-zones of dead-zone and discontinuous nonlinearity, correspondingly.

It is easy to separate the particles of $\tilde{y}(k)$ by choosing the middle data set $\mathbf{Y}_{2}$ in the case of saturation, or the left-hand side and right-hand side sets $\mathbf{Y}_{1}, \mathbf{Y}_{3}$ in the cases of a dead-zone and a discontinuous nonlinearity, respectively, if the measurement noise $e(k)$ is absent. In such a case, only these corresponding data particles are needed for further extraction of the unmeasurable internal signal $x(k)$. Note that, for saturation there exists a regime where true values of $y(k)$ are assured. In such a case, they are equal to respective values of the unmeasurable intermediate signal $x(k)$. 


\section{Internal Signal Extraction}

To estimate the parameters $\beta$ of the FIR model (5), one can use the expression of the form

$$
\hat{\beta}=\left(\boldsymbol{\Lambda}_{2}^{T} \boldsymbol{\Lambda}_{2}\right)^{-1} \boldsymbol{\Lambda}_{2}^{T} \mathbf{Y}_{2},
$$

for the Wiener system with the saturation (16) or that of the form

$$
\hat{\beta}=\left(\tilde{\boldsymbol{\Lambda}}_{4}^{T} \tilde{\boldsymbol{\Lambda}}_{4}\right)^{-1} \tilde{\boldsymbol{\Lambda}}_{4}^{T} \tilde{\mathbf{Y}}_{4},
$$

for the dead-zone (17) and discontinuous nonlinearity (20)-(23). Here

$$
\hat{\beta}^{T}=\left(\hat{\beta}_{0}, \hat{\beta}_{1}, \ldots, \hat{\beta}_{\nu}\right)
$$

is a $(\nu+1) \times 1$ vector of the estimates of parameters $(9)$;

$$
\tilde{\mathbf{Y}}_{4}=\left[\begin{array}{l}
\mathbf{Y}_{1} \\
\mathbf{Y}_{3}
\end{array}\right]
$$

and

$$
\tilde{\Lambda}_{4}=\left[\begin{array}{l}
\Lambda_{1} \\
\Lambda_{3}
\end{array}\right]
$$

Note that, all proofs based on the deterministic regression matrix are valid here as well because the interchange of equations does not influence the accuracy of LS estimates to be calculated.

Then the estimate $\hat{x}(k)$ of the intermediate signal $x(k)$ could be extracted using (5) where true values (9) are replaced by their estimates $\hat{\beta}$, calculated by (24) or (25), i.e.,

$$
\hat{x}(k)=\hat{\beta}_{0}+\hat{\beta}_{1} u(k)+\hat{\beta}_{2} u(k-1)+\cdots+\hat{\beta}_{\nu} u(k-\nu+1),
$$

$\forall k \in \overline{\nu, N}$, respectively. Thus, the result of this step is the auxiliary signal $\hat{x}(k)$ that is a reconstructed version of the intermediate unmeasurable one $x(k)$. It will be used to calculate the LS estimate $\hat{\boldsymbol{\Theta}}$ of the vector of parameters $\boldsymbol{\Theta}^{T}=\left(b_{1}, \ldots, b_{m}, a_{1}, \ldots, a_{m}\right)$ of the linear block (1) (Fig. 1) by the well-known formula (7)

$$
\hat{\mathbf{\Theta}}=\left(\mathbf{X}^{T} \mathbf{X}\right)^{-1} \mathbf{X}^{T} \mathbf{Y} .
$$

Here $\hat{\boldsymbol{\Theta}}^{T}=\left(\hat{b}_{1}, \ldots, \hat{b}_{m}, \hat{a}_{1}, \ldots, \hat{a}_{m}\right)$ is $2 m \times 1$ is the estimate of the vector of parameters $\boldsymbol{\Theta}$,

$$
\mathbf{X}=\left[\begin{array}{cccccc}
u(m+\nu) & \ldots & u(\nu+1) & -\hat{x}(m+\nu) & \ldots & -\hat{x}(\nu+1) \\
u(m+\nu+1) & \ldots & u(\nu+2) & -\hat{x}(m+\nu+1) & \ldots & -\hat{x}(\nu+2) \\
\vdots & & \vdots & \vdots & & \vdots \\
u(N-1) & \ldots & u(N-m) & -\hat{x}(N-1) & \ldots & -\hat{x}(N-m)
\end{array}\right]
$$


is the $(N-m-\nu) \times 2 m$ matrix, consisting of observations of the input $u(k)$ and the auxiliary signal $\hat{x}(k)(29), \mathbf{Y}$ is the $(N-m-\nu) \times 1$ vector of output observations.

Then, the second, and the main estimate $\tilde{x}(k) \forall k \in \overline{1, N}$ of the internal signal is calculated by

$$
\tilde{x}(k)=G\left(q^{-1}, \hat{\mathbf{\Theta}}\right) u(k)=\frac{\hat{b}_{1} q^{-1}+\cdots+\hat{b}_{m} q^{-m}}{1+\hat{a}_{1} q^{-1}+\cdots+\hat{a}_{m} q^{-m}} u(k),
$$

that can be compared with its previous version (29), based on the FIR model.

Sometimes, in the presence of the measurement noise $e(k) \forall k \in \overline{1, N}$ we cannot accurately determine to which of the three regimes given an observation belongs. The wrong attribution of observations to any of the given regime is likely to yield an inaccuracy in the extraction of the internal signal (29) as well as in the estimation of unknown parameters $\Theta$. Thus, a question arises to which regime the observation belongs at the boundary of the respective data set in a noisy environment. Such a problem could be solved if one rewrites the middle data set (11) as follows:

$$
\mathbf{Y}_{\mathbf{2}}=\left[\begin{array}{c}
\mathbf{Y}_{21} \\
\mathbf{Y}_{22} \\
\mathbf{Y}_{23}
\end{array}\right]=\boldsymbol{\Lambda}_{2} \beta=\left[\begin{array}{c}
\boldsymbol{\Lambda}_{21} \\
\boldsymbol{\Lambda}_{22} \\
\boldsymbol{\Lambda}_{23}
\end{array}\right] \beta
$$

Assuming that the hard nonlinearity damages about 10 percent of observations of $y(k) \forall k \in \overline{1, N}$ the rearranged data contained in the vector $\mathbf{Y}_{22}$ should be chosen from the interval with a lower interval bound slightly higher than 5 percent and the upper interval bound slightly lower than 95 per cent of the sampled reordered observations of $\tilde{y}(k)$. Thus, both vectors $\mathbf{Y}_{21}$ and $\mathbf{Y}_{23}$ will contain approximately 10 percent of observations of $\tilde{y}(k)$ that one could attribute to the left-hand or right-hand data sets, respectively. Such observations ought to be rejected, and only the observations that belong to the true middle data set $\mathbf{Y}_{22}$ ought to be processed. Then expression (24) turns out to be of the form

$$
\hat{\beta}=\left(\boldsymbol{\Lambda}_{22}^{T} \boldsymbol{\Lambda}_{22}\right)^{-1} \boldsymbol{\Lambda}_{22}^{T} \mathbf{Y}_{22} .
$$

For a discontinuous-nonlinearity (20)-(23) one can also rewrite $\tilde{\mathbf{Y}}_{4}$ in (25) according to

$$
\tilde{\mathbf{Y}}_{\mathbf{4}}=\left[\begin{array}{c}
\mathbf{Y}_{11} \\
\mathbf{Y}_{12} \\
\mathbf{Y}_{31} \\
\mathbf{Y}_{32}
\end{array}\right]=\tilde{\boldsymbol{\Lambda}}_{\mathbf{4}} \beta=\left[\begin{array}{c}
\boldsymbol{\Lambda}_{11} \\
\boldsymbol{\Lambda}_{12} \\
\boldsymbol{\Lambda}_{31} \\
\boldsymbol{\Lambda}_{32}
\end{array}\right] \beta .
$$

Let us now rearrange the vector $\tilde{\mathbf{Y}}_{4}$ as follows

$$
\tilde{\mathbf{Y}}_{4}=\left[\begin{array}{l}
\mathbf{Y}_{11} \\
\mathbf{Y}_{32}
\end{array}\right]
$$


assuming that both vectors $\mathbf{Y}_{12}$ and $\mathbf{Y}_{31}$, to be rejected, will contain approximately 10 percent of observations of $\tilde{y}(k)$ attributed to the middle data set, respectively. In such a case, the matrix $\tilde{\Lambda}_{4}$ is

$$
\tilde{\boldsymbol{\Lambda}}_{\mathbf{4}}=\left[\begin{array}{l}
\boldsymbol{\Lambda}_{11} \\
\boldsymbol{\Lambda}_{32}
\end{array}\right]
$$

Afterwards $\hat{\beta}$ is calculated according to (25). Note that, before the estimation of parameters of the FIR filter (29), one has to sort out correct samples from the initial set of rearranged output observations rejecting some incorrect data portions, if the available hard-nonlinearity is a saturation or dead-zone. For the preload and backlash a complete set of data could be used, because both nonlinearities do not cut off observations $y(k) \forall k=\overline{1, N}$. Moreover, in (7) instead of $y(k)$ the values $y(k)+\hat{a} \operatorname{sign}(y(k))$ or $y(k)-\hat{a} \operatorname{sign}(y(k)) k=1,2, \ldots, N$ ought to be substituted, if the hard-nonlinearity is the preload. The respective abovementioned values are needed if the hard-nonlinearity is the dead-zone, too. In such a case, instead of ordinary rearranged $y(k)$ meanings available in the right- and left-data sets, they ought to be substituted, respectively. Here $\hat{a}$ is an initial value of a threshold $a$ to be chosen in advance. For the backlash the initial version $\hat{x}(k)$ of the internal signal has to be available. Then, in (29) instead of $y(k)$ the value of $y(k)+\hat{a}$, if $\hat{x}(k)-\hat{x}(k-1)>0$ or $y(k)-\hat{a}$, if $\hat{x}(k)-\hat{x}(k-1)<0$, or $\hat{x}(k-1)$, if $\hat{x}(k)=\hat{x}(k-1)$ ought to be replaced according to (19).

The estimate of the signal $\hat{x}(k) \forall k \in \overline{1, N}$ can be determined using the technique, based on the iterative procedure, consisting of two steps. In the first step, the estimate of the internal signal $\hat{x}(k) \forall k \in \overline{1, N}$ is calculated, given the available data $(u(k), y(k)) \forall k \in \overline{1, N}$, and the estimates $\hat{\beta}$. In the second step, $\hat{x}(k) \forall k \in \overline{1, N}$ is used to recalculate the estimates of parameters $\beta$. The technique to be used for the saturation could be explained by the following steps: (a) cut-off observations of $y(k)$ could be rejected beforehand; (b) the LS problem (24), consisting of the identification of parameters of the FIR filter (5) has been solved using the middle data set; (c) the estimate of the internal signal has been calculated by (24), using the estimates of parameters of the FIR filter that have been calculated in (b); (d) the LS problem (30), consisting of the identification of parameters of the linear block (1) has been solved using the matrix (31); (e) return to (b). Iterations of the above mentioned calculations are continued until the convergence is assured.

\section{Simulation Results}

We consider here the common examples of hard-nonlinearities in a control system, such as the saturation, preload, dead-zone, and backlash (Fig. 2), and discontinuous nonlinearity (Fig. 3), too. The true intermediate signal of the control system is given by

$$
x(k)=\frac{q^{-1}}{\left(1-0.7 q^{-1}\right)^{2}} u(k),
$$




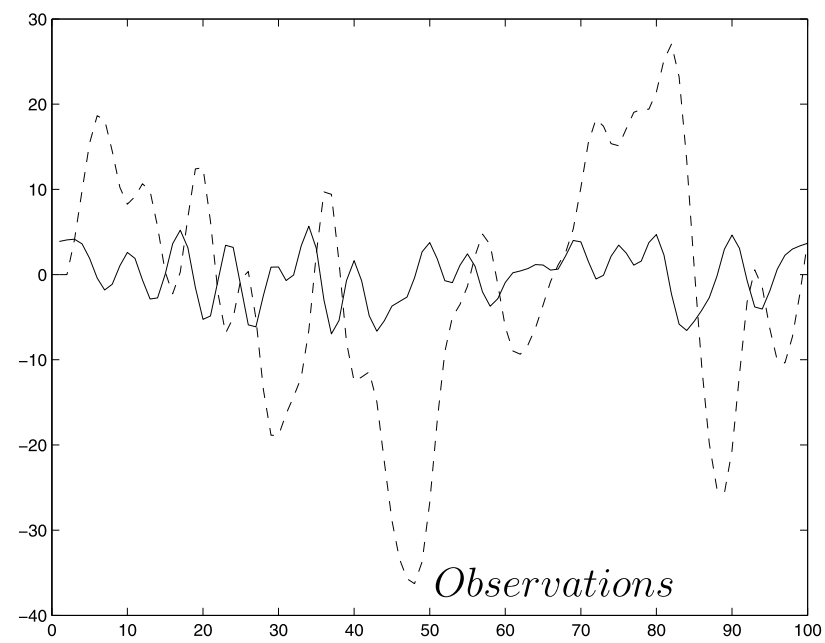

Fig. 4. Signals of the simulated linear system (38): input $u(k) \forall k \in \overline{1, N}$ is a continuous line; internal signal $x(k) \forall k \in \overline{1, N}$ is a dashed line.

with the sum of sinusoids

$$
u(k)=\sum_{l=1}^{20} \sin \left(k \pi l / 10+\varphi_{l}\right),
$$

$\forall k=\overline{1, N}$, having the stochastic phases $\varphi_{l} \forall l=\overline{1,20}$ with a uniform distribution on $[0,2 \pi]$, as an input to the linear block (1). Firstly, four output signals were generated: for the saturation, for the dead-zone, for the preload, and for the backlash by formulas (16)-(19), respectively. Thus, for each of the four simulated control system outputs (16)-(19) (see Fig. 5) the internal signal $x(k) \forall k \in \overline{1, N}$ is the same (Fig. 4), and as shown in (Fig. 5), for each of hard-nonlinearities, too.

$N=100$ data points were generated, without additive process and measurement noises (Fig. 6), and with them (Fig. 7) according to $x(k)=x(k)+\lambda_{1} v(k) \forall k \in$ $\overline{1, N}, y(k)=y(k)+\lambda_{2} e(k) \forall k \in \overline{1, N}$, respectively. Here $\lambda_{1}, \lambda_{2}$ were chosen such that SNRs (the square root of the ratio of signal and noise variances) were equal to 100 for the process noise $v(k) \forall k \in \overline{1, N}$, and to 50 for the measurement noise $e(k) \forall k \in \overline{1, N}$. Further $S N R^{v}$ and $S N R^{e}$ will be used for process and measurement noises, respectively. Next, it followed parametric identification of FIR model (5), and the recursive extraction of the internal signal by (29). Note that for the saturation and the dead-zone, 60 cut-off rearranged output $y(k)$ observations were rejected beforehand, and only 40 observations were processed in both cases by means of techniques described in the fourth chapter. It could be mentioned that we also do not use the first 14 observations of $y(k)$ because it is important to fill the matrix (8) and the vector (7) completely, thus avoiding the influence of initial conditions. The simulation results imply that the accuracy of extracted versions of the internal sig- 

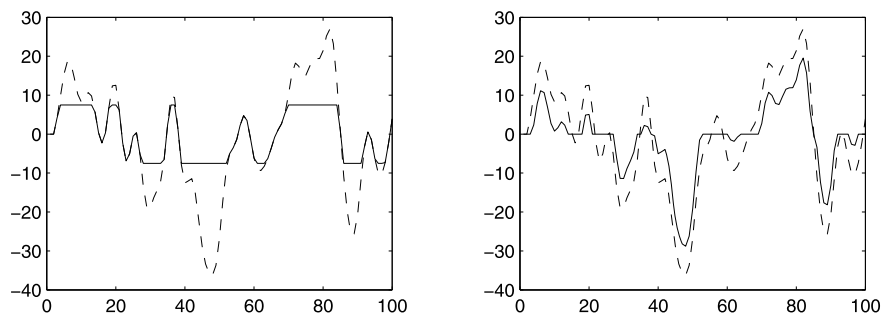

$a$

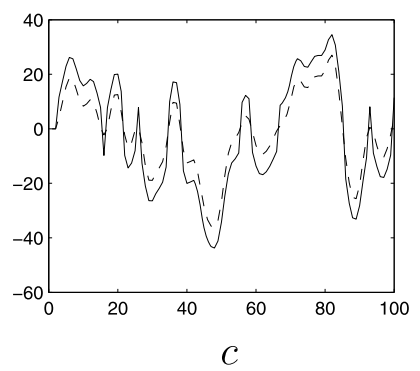

b

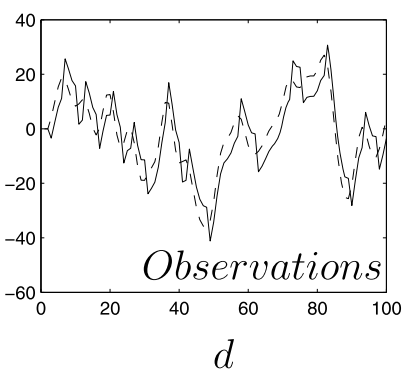

Fig. 5. Signals of the simulated system (38): output $y(k) \forall k \in \overline{1, N}$ is a continuous line, the internal signal $x(k) \forall k \in \overline{1, N}$ is a dashed line. The hard-nonlinearity: saturation (a), dead-zone (b), preload (c), backlash (d). The threshold $a=7.5$.
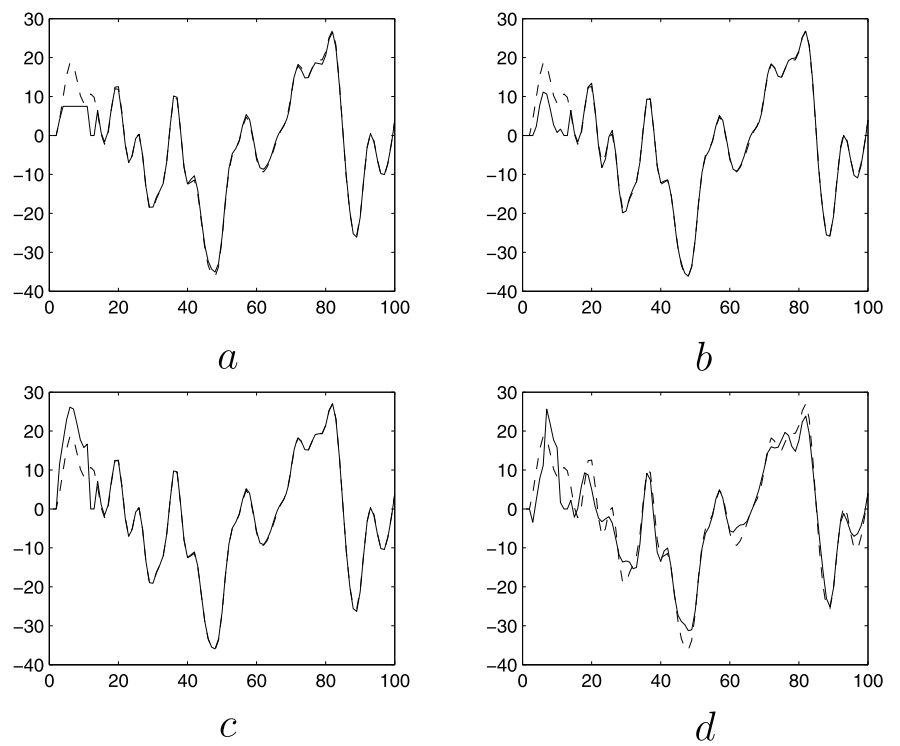

Fig. 6. Signals of the simulated system (38): reconstructed according the formula (29) internal signal $\hat{x}(k) \forall k \in \overline{1, N}$ is a continuous line, true internal signal $x(k) \forall k \in \overline{1, N}$ calculated by (38) is a dashed line. The hard-nonlinearity: saturation (a), dead-zone (b), preload (c), backlash (d). 

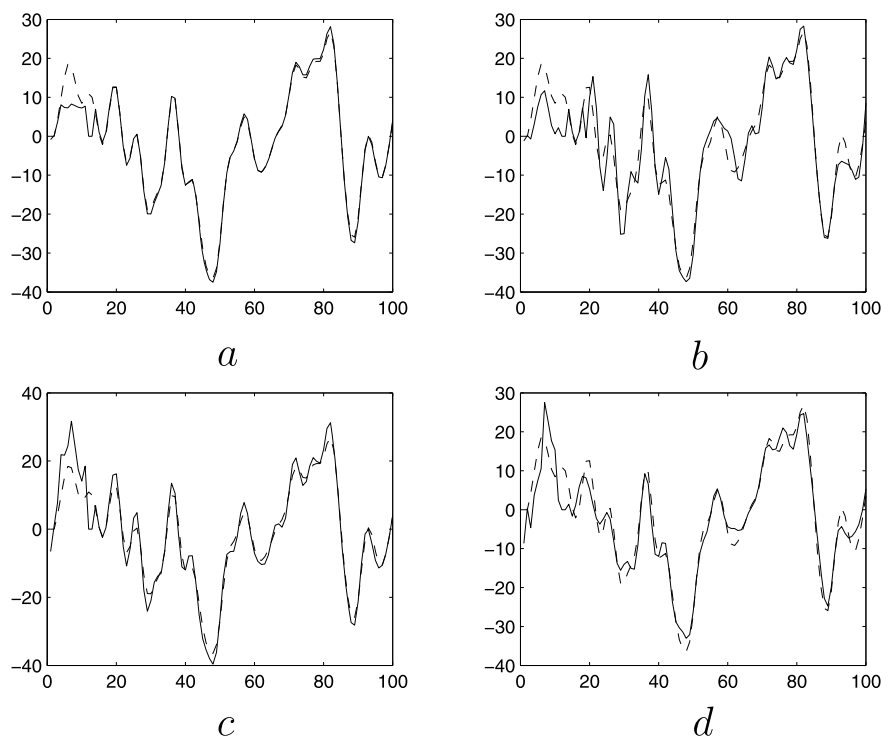

Fig. 7. The values and markings are the same as in Fig. 6 except that signals are observed in a noisy environment.

nal $x(k) \forall k \in \overline{1, N}$, calculated for the saturation, dead-zone and preload, is higher as compared to the accuracy of the same signal reconstructed version, obtained for the backlash with the exception of the transition process for the FIR model. Note that for the backlash, it is important to have the initial version of the internal signal $x(k) \forall k \in \overline{1, N}$ close enough to the true one. The main estimates of the internal signal also were calculated by formula (32) for each hard-nonlinearity. They were compared with the previous respective versions (29). It was concluded that the results were similar enough.

For a discontinuous nonlinearity $N=100$ data points have been generated, without additive process and measurement noises (see Fig. 8a), too. The output $y(k)$ was calculated according to (20)-(23) with the following values of parameters:

$$
m_{1}=1, \quad m_{2}=1.5, \quad D=1.25, \quad b=0.3,
$$

for the nonlinear block. First of all the LS problem, consisting in the estimation of parameters of the FIR filter (5), was solved. The whole number of its parameters $\nu=14$ was chosen. The initial values of respective constants were determined as follows:

$$
\hat{m}_{1}=0.1, \quad \hat{m}_{2}=0.15, \quad \hat{D}=0.125, \quad \hat{b}=0.03 .
$$



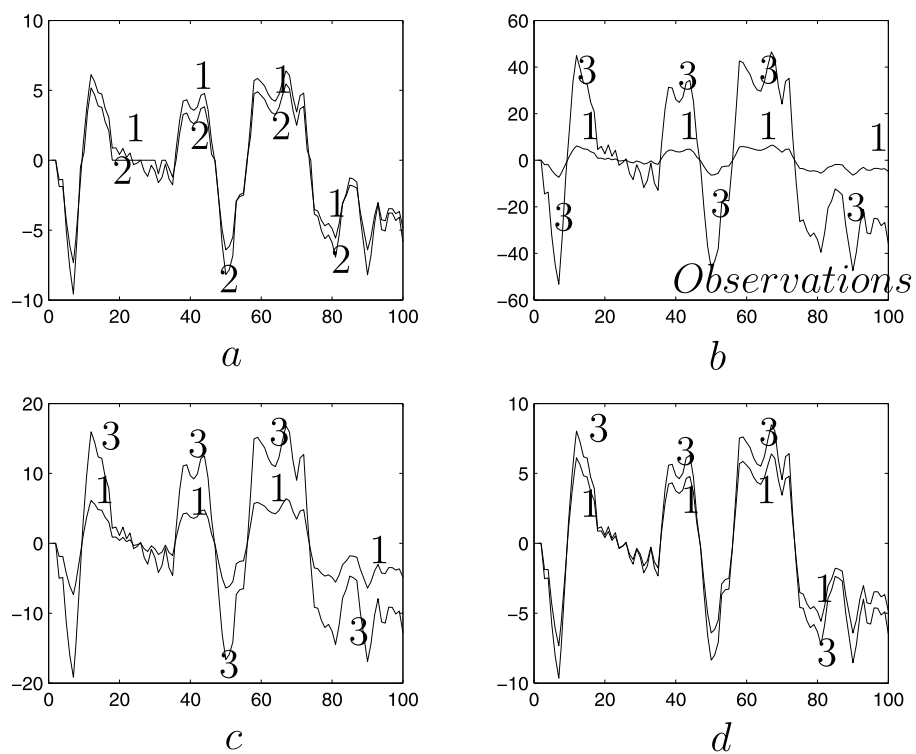

Fig. 8. Signals $x(k)$ and $y(k)$, and the extracted $x(k)$ versions depending on the number (a) of observations as well as on that of iterations (b)-(d). Signals: $x(k), y(k)$ (a), $x(k), \hat{x}_{1}(k)(\mathrm{b}), x(k), \hat{x}_{2}(k)(\mathrm{c}), x(k), \hat{x}_{3}(k)$ (d). Curves: $x(k)(1), y(k)(2), \hat{x}_{1}(k), \hat{x}_{2}(k), \hat{x}_{3}(k)$ (3). Iterations: 1 (b), 10 (c), 20 (d).

In such a case, 19 cut-off observations of $y(k)$ from 100 initial ones were rejected beforehand. Note that like for other hard-nonlinearities we do not use here the first 14 observations of $y(k)$. The estimates of FIR filter parameters were calculated using $30 \mathrm{ob}$ servations

$$
\tilde{x}(k)=\hat{m}_{1}^{-1} y(k)+\hat{D} \operatorname{sgn}(y(k))-\hat{b} \hat{m}_{1}^{-1} \operatorname{sign}(y(k))
$$

as $y(k)>0$, and 37 observations

$$
\tilde{x}(k)=\hat{m}_{2}^{-1} y(k)+\hat{D} \operatorname{sgn}(y(k))-\hat{b} \hat{m}_{2}^{-1} \operatorname{sign}(y(k))
$$

with $y(k)<0$. They were substituted instead of the respective values of $y(k)$ in (7). Later on, the internal signal $x(k)$ was reconstructed according to (29), using the estimates of the FIR filter parameters that were calculated beforehand. Thus, the signal $\hat{x}(j) \forall j=\overline{15,100}$ has been determined. The estimates $\hat{b}_{1}, \hat{a}_{1}, \hat{a}_{2}$ of parameters $b_{1}, a_{1}, a_{2}$ of the control system (38):

$$
\hat{b}_{1}=1, \quad \hat{a}_{1}=-1.4, \quad \hat{a}_{2}=0.49
$$

have been calculated at the next step, and they have been used to calculate the second estimate (32) of the intermediate internal signal $\tilde{x}(k) \forall k=\overline{3,100}$. Estimates of the 
parameters $m_{1}, m_{2}$ and $D, b$ have been calculated as follows (Malinvaud, 1969):

$$
\begin{aligned}
& \hat{m}_{1}=\frac{\sum_{j=3}^{\hat{N}_{1}} y(j) \tilde{x}(j)}{\sum_{j=3}^{\hat{N}_{1}} \tilde{x}^{2}(j)}, \quad \text { for } y(j)>0 \forall j=\overline{1, \hat{N}_{1}}, \\
& \hat{m}_{2}=\frac{\sum_{j=3}^{\hat{N}_{2}} y(j) \tilde{x}(j)}{\sum_{j=3}^{\hat{N}_{2}} \tilde{x}^{2}(j)}, \quad \text { for } y(j)<0 \forall j=\overline{1, \hat{N}_{2}}, \quad \text { and } \\
& \hat{D}=\frac{1}{\hat{m}_{2}-\hat{m}_{1}}\left\{\frac{1}{\hat{N}_{1}} \sum_{j=3}^{\hat{N}_{1}}\left[y(j)-\hat{m}_{1} \tilde{x}(j)\right]+\frac{1}{\hat{N}_{2}} \sum_{j=3}^{\hat{N}_{2}}\left[y(j)-\hat{m}_{2} \tilde{x}(j)\right]\right\}, \\
& \hat{b}=\frac{1}{2}\left\{\frac{1}{\hat{N}_{1}} \sum_{j=3}^{\hat{N}_{1}}\left[y(j)-\hat{m}_{1} \tilde{x}(j)\right]-\frac{1}{\hat{N}_{2}} \sum_{j=3}^{\hat{N}_{2}}\left[y(j)-\hat{m}_{2} \tilde{x}(j)\right]\right. \\
& \left.\quad+\left(\hat{m}_{1}+\hat{m}_{2}\right) \hat{D}\right\}
\end{aligned}
$$

respectively. Here $\hat{N}_{1}=30$ and $\hat{N}_{2}=37$ were used. The iterations of the calculations have been repeated for several times. Note that, during the previous calculations, the estimates $\hat{b}_{1}, \hat{a}_{1}, \hat{a}_{2}$ of parameters $b_{1}, a_{1}, a_{2}$ of the linear block (1) and estimates $\hat{m}_{1}, \hat{m}_{2}, \hat{D}, \hat{b}$ of parameters $m_{1}, m_{2}, D, b$ of the discontinuous nonlinearity (20)-(23), that were determined in the previous iteration, have been used in the current iteration, respectively.

The intermediate signal $x(k)$, the output signal $y(k)$, and three reconstructed versions $\hat{x}_{1}(k), \hat{x}_{2}(k)$ and $\hat{x}_{3}(k)$ of the second estimate $\tilde{x}(k)$ of the internal signal $x(k)$ that have been determined after executing a different number of iterations $(1,10,20)$, are shown in Fig. 8. Note that, the signal $\hat{x}_{1}(k)$ (curve 3 in Fig. 8b),extracted in the first iteration, is the worst estimate of internal $x(k)$ in comparison with the signals $\hat{x}_{2}(t)$, $\hat{x}_{3}(t)$ (curves 3 in Figs. 8c, 8d). It follows that by increasing the number of iterations, the accuracy of reconstruction of the true signal $x(k)$ has been increased, too. Therefore, it suffices to perform approximately 30 iterations in order to determine the estimate $\hat{x}(k)$ that accurately enough approximates the true $x(k)$. In Fig. 9a input and output signals are shown. A complete set of the output data is presented here, too. Really, a reduced set (67 observations) was used during the intermediate signal extraction and the parametric estimation. The estimates $\hat{b}_{1}, \hat{a}_{1}, \hat{a}_{2}$ of parameters $b_{1}, a_{1}, a_{2}$ depending on the numbers of iterations are shown in Figs. 9b-9d. It is easy to understand that deviation of estimates from the true values is not significant. On the other hand, the estimates $\hat{a}_{1}, \hat{a}_{2}$ do not approach their true values with a remarkable increase of the iteration number. In Fig. 10 , the estimates $\hat{m}_{1}, \hat{m}_{2}, \hat{D}, \hat{b}$ of parameters $m_{1}, m_{2}, D, b$ are presented depending on the number of iterations of the calculations performed. In such a case, the transition process of convergence of the estimates $\hat{b}_{1}, \hat{a}_{1}, \hat{a}_{2}$ and $\hat{m}_{1}, \hat{m}_{2}, \hat{D}, \hat{b}$ to the true values $b_{1}, a_{1}, a_{2}$ and $m_{1}, m_{2}, D, b$, respectively, is over, too. In Figs. 11-13, 


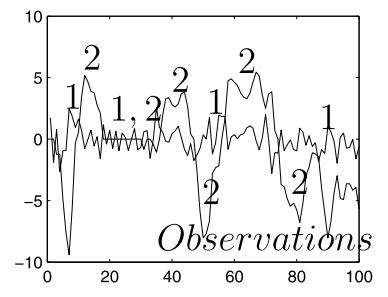

$a$

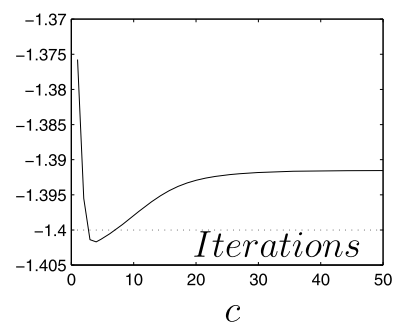

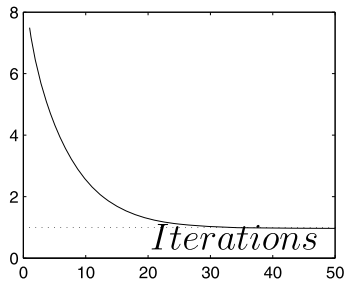

$b$

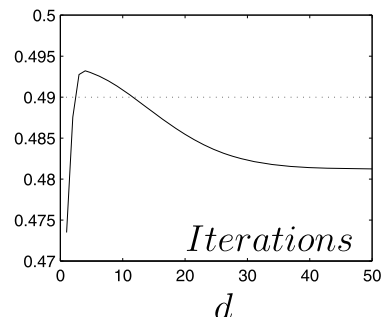

Fig. 9. Signals: $u(k)(1), y(k)(2)$ in (a), and estimates $\hat{b}_{1}(\mathrm{~b}), \hat{a}_{1}$ (c), $\hat{a}_{2}$ (d) of parameters $b_{1}, a_{1}, a_{2}$ (b)-(d) (dotted lines) depending on observations (a), as well as on iterations (b)-(d).

the same values are shown as in Figs. 8-10, respectively, but almost twice larger sets of observations as the previous ones. The accuracy of $\hat{x}(k)$ and estimates $\hat{b}_{1}, \hat{a}_{1}, \hat{a}_{2}$ and $\hat{m}_{1}, \hat{m}_{2}, \hat{D}, \hat{b}$ changes slightly, when the number of processed observations is increased (see Fig. 11-13) - only the estimate $\hat{b}$ of the preload constant $b$ (Fig. 13d) is more accurate than that calculated by processing 67 samples (Fig. 10d). Note that, actually, in such a case, only 130 observations of the input-output data have been processed by the proposed iterative technique. As much as 70 observations from 200 have been rejected as cut-off observations that had been damaged while passing through the discontinuous nonlinearity.

The estimation results shown in Figs. 14-19 are determined in the presence of the additive measurement noise (Figs. 14-17), as well as in the presence of both process and measurement noises (Figs. 18-19). Note that, in parts b, c, d of (Fig. 18), and in all the parts of (Fig. 19) the respective estimates are presented in ten curves. The additive measurement noise, corrupting $y(k)$, affects $\hat{x}(k)$, as well as the estimates $\hat{b}_{1}, \hat{a}_{1}, \hat{a}_{2}$ and $\hat{m}_{1}, \hat{m}_{2}, \hat{D}, \hat{b}$ (Figs. 14-17). It seems that $\hat{b}$ does not approach the true $b$ (Fig. 17d) for such two realizations of process and measurement noises, correspondingly. Figs. 20 and 21 illustrate the estimates $\hat{b}_{1}, \hat{a}_{1}, \hat{a}_{2}$ and $\hat{m}_{1}, \hat{m}_{2}, \hat{D}, \hat{b}$, that are averaged by 10 experiments. We can assert that the estimates $\hat{b}_{1}, \hat{a}_{1}, \hat{a}_{2}$ and $\hat{m}_{1}, \hat{m}_{2}, \hat{D}, \hat{b}$ approach the true values in the sense of mean squares, respectively, after the number of different realizations of noisy input-output data, used to estimate the parameters $b_{1}, a_{1}, a_{2}$ and $m_{1}, m_{2}, D, b$ (Figs. 18-21), has been increased. 

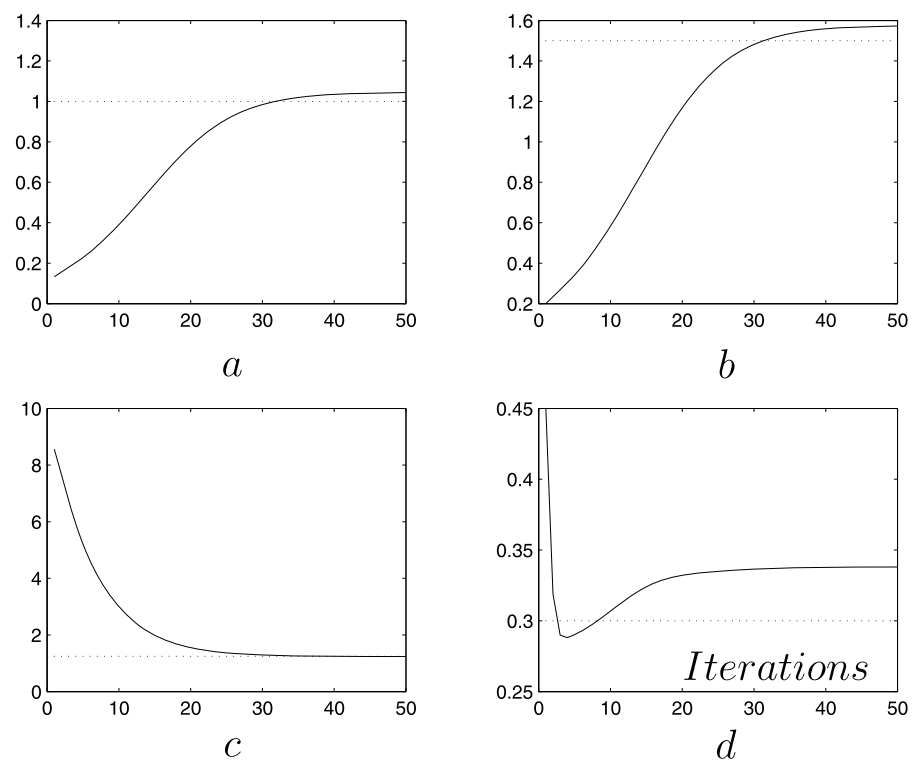

Fig. 10. Estimates $\hat{m}_{1}$ (a), $\hat{m}_{2}$ (b), $\hat{D}$ (c), $\hat{b}$ (d) of the true values of $m_{1}, m_{2}, D, b$ (dotted lines) of the nonlinearity (20)-(23).
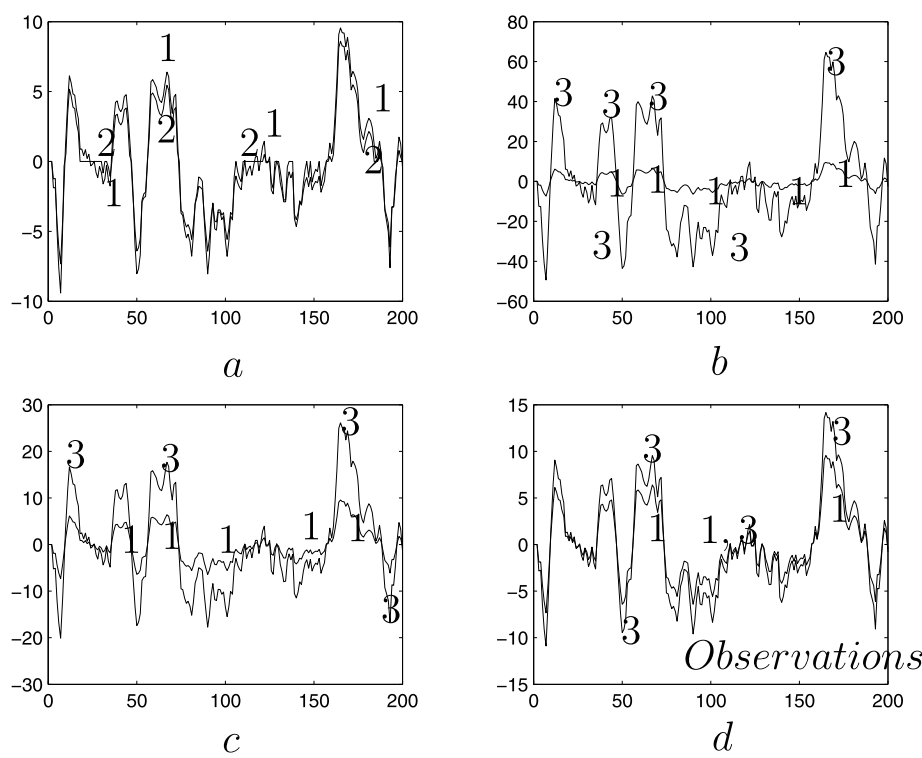

Fig. 11. Values and markings are the same as in Fig. 8. 

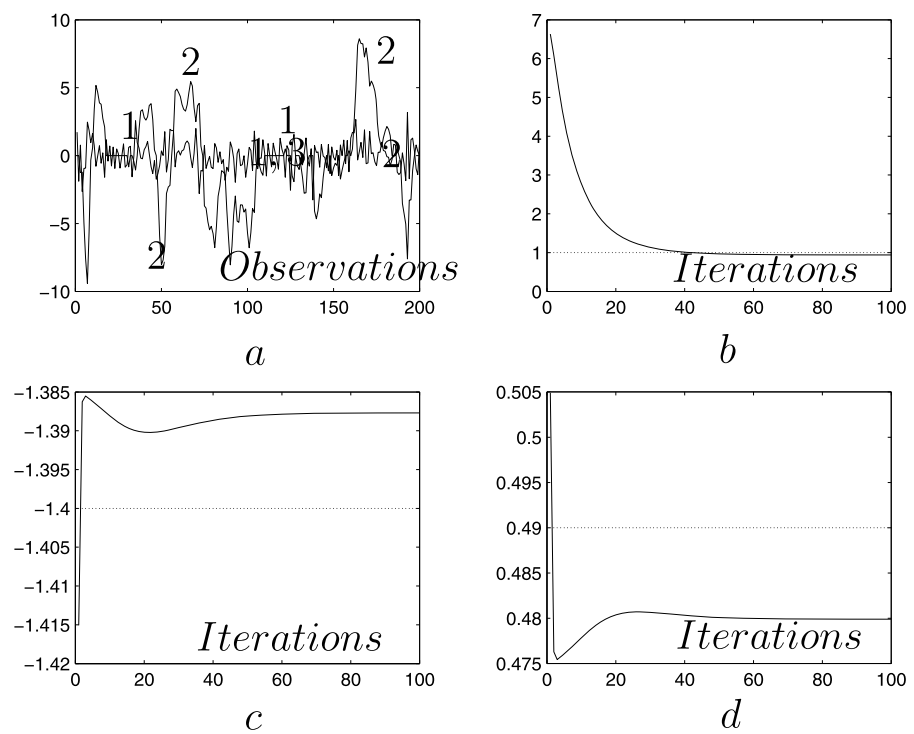

Fig. 12. Values and markings are the same as in Fig. 9.
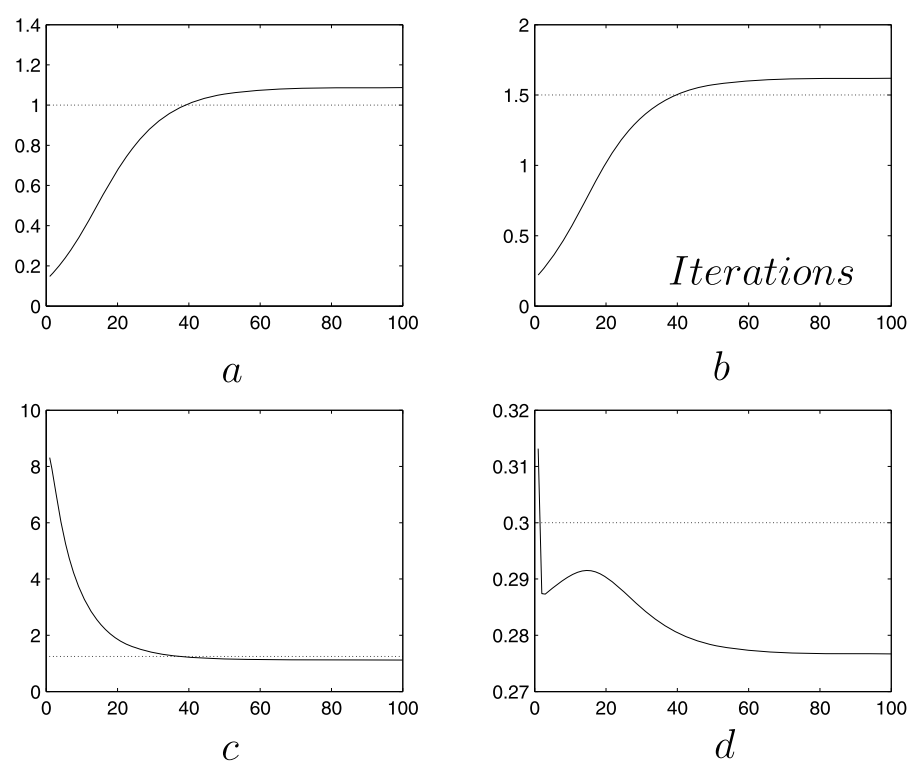

Fig. 13. Values and markings are the same as in Fig. 10. 

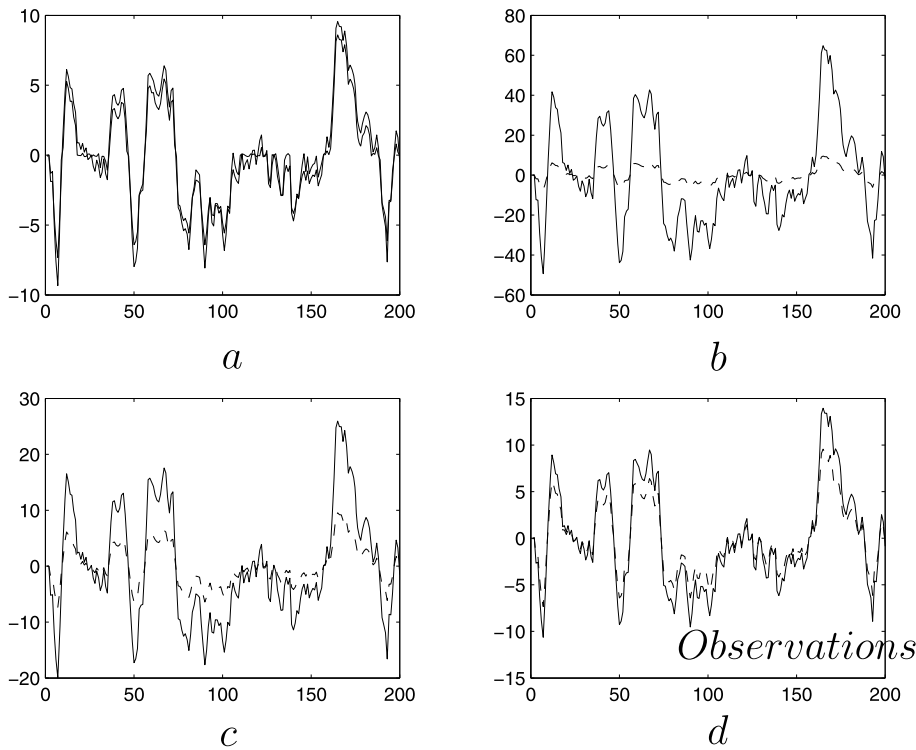

Fig. 14. Values and markings are the same as in Fig. 8. $\mathrm{SNR}^{e}=100$
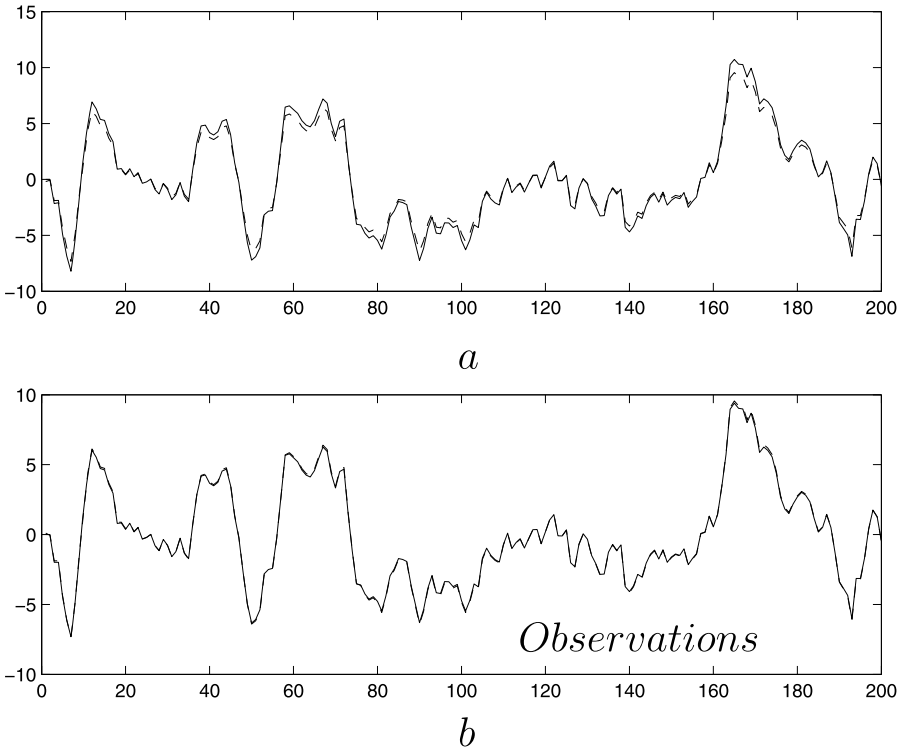

Fig. 15. The internal signal $x(k)$ and $\hat{x}(k)$. Iterations: 30 (a), 40 (b). Curves: continuous is $\hat{x}(k)$, dashed is $x(k) . \mathrm{SNR}^{e}=100$ 

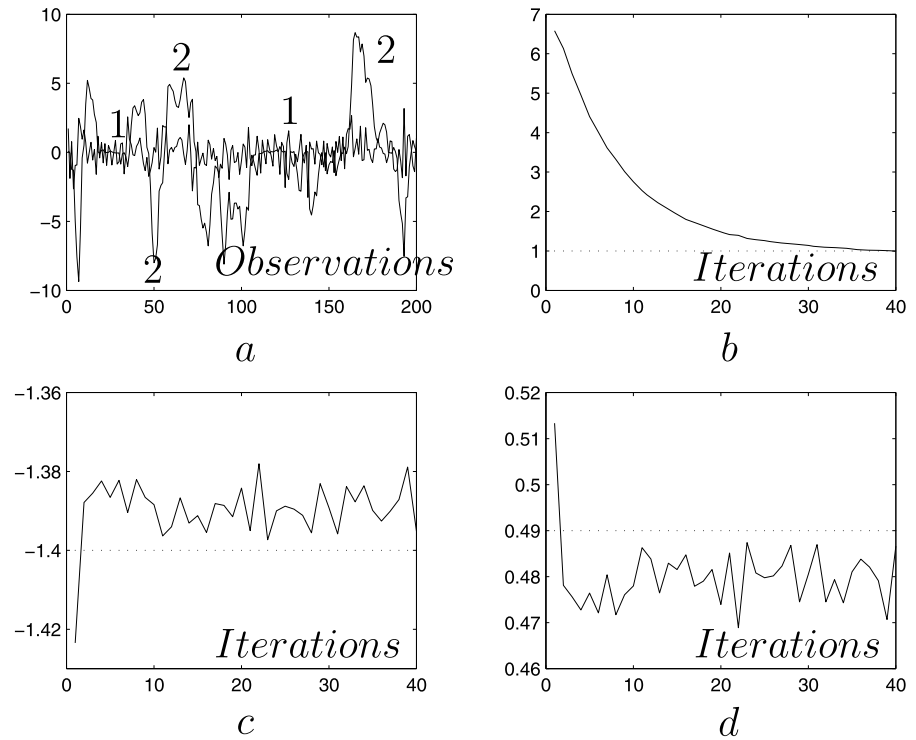

Fig. 16. Values and markings are the same as in Fig. 9. $\mathrm{SNR}^{e}=100$.
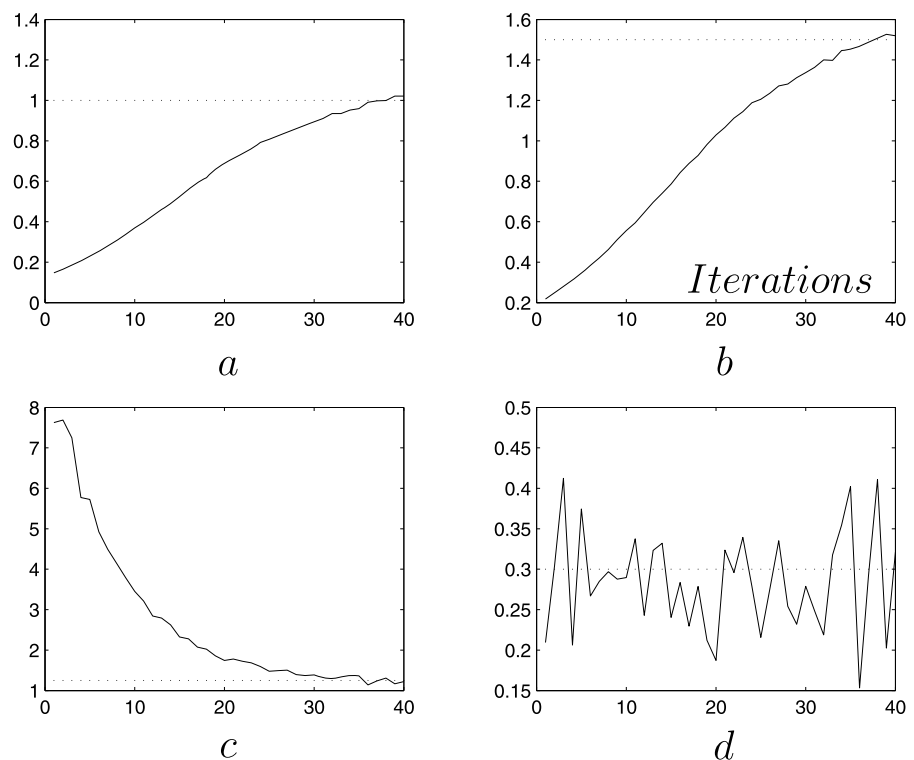

Fig. 17. Values and markings are the same as in Fig. 10. $\mathrm{SNR}^{e}=100$. 

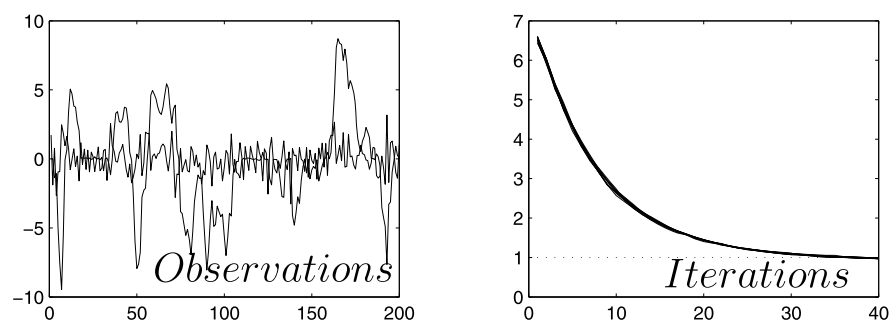

$a$

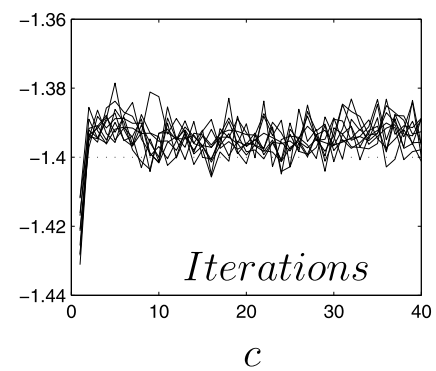

$b$

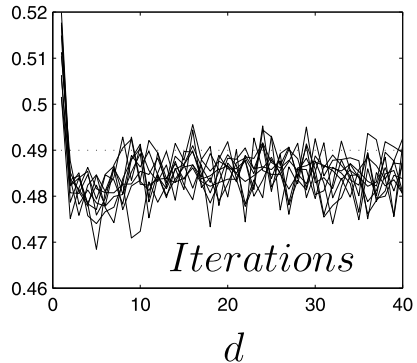

Fig. 18. Values and markings are the same as in Fig. 16 except the number of curves in parts (b)-(d), where the estimates of $b_{1}, a_{1}, a_{2}$ depending on the number of iterations are shown in ten, respectively. $\mathrm{SNR}^{v}=100, S N R^{e}=50$.

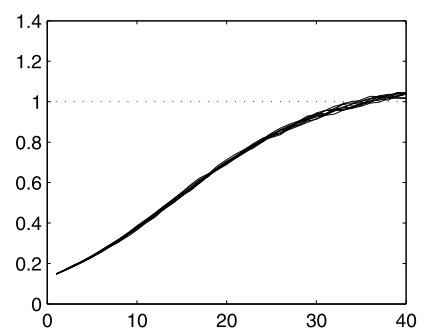

$a$

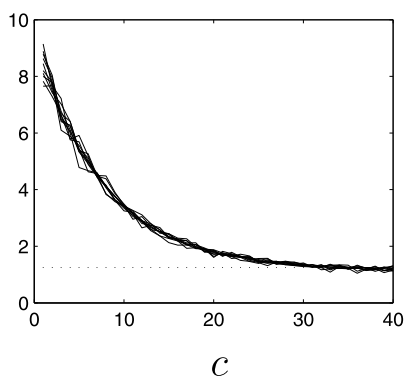

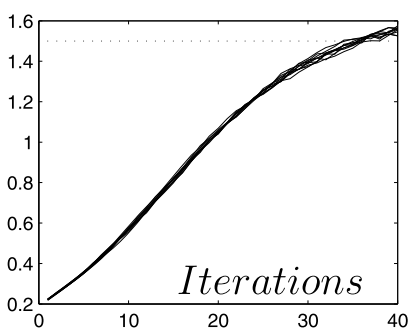

$b$

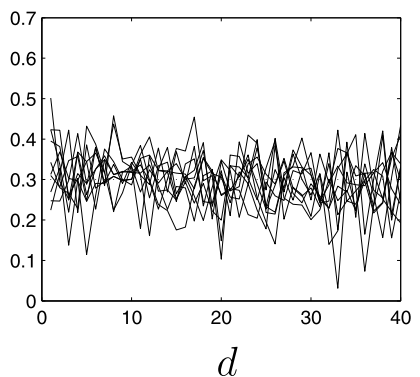

Fig. 19. Values and markings are the same as in Fig. 17 except the number of curves, where estimates of $m_{1}, m_{2}, D, b$ depending on the number of iterations are shown in ten. $\mathrm{SNR}^{v}=100, \mathrm{SNR}^{e}=50$. 

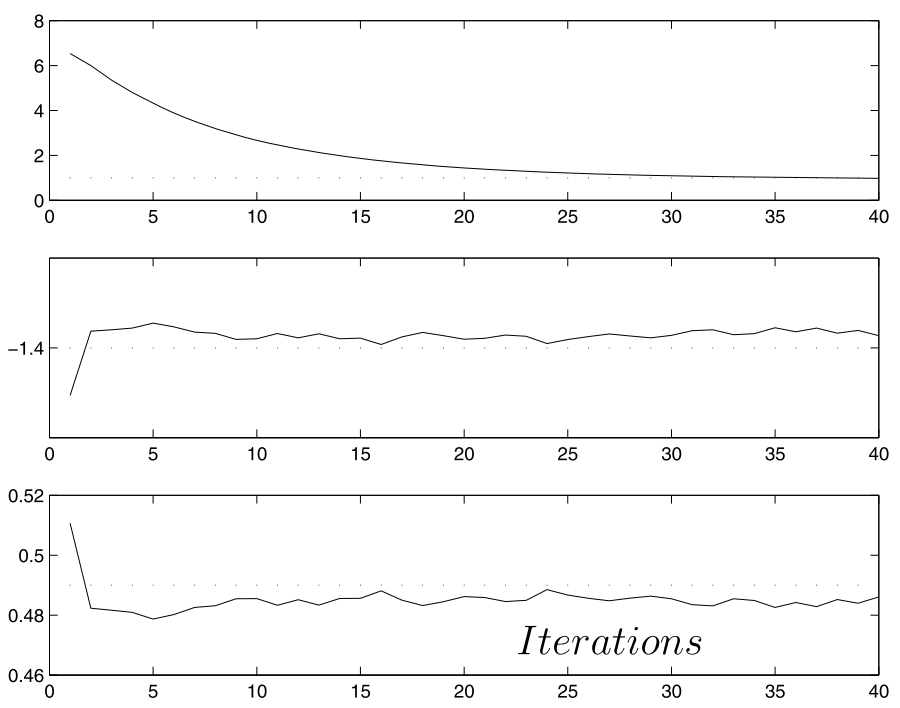

Fig. 20. Averaged estimates of the values shown in Figs. 18b, 18c, 18d. Parts: $\hat{b}_{1}$ (top), $\hat{a}_{1}$ (middle), $\hat{a}_{2}$ (bottom).
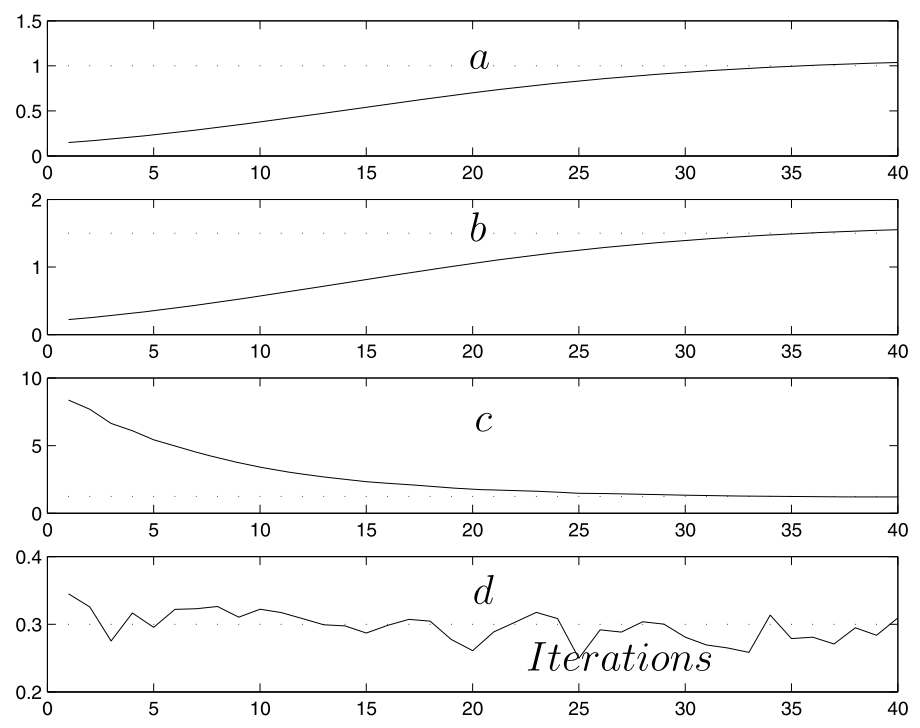

Fig. 21. Averaged estimates of values shown in Fig. 19. Parts: $\hat{m}_{1}$ (a), $\hat{m}_{2}$ (b), $\hat{D}$ (c), $\hat{b}$ (d).

\section{Conclusions}

In control systems hard-nonlinearities can occur either in the control actuators or in measurement devices. There exist intelligent control approaches for the compensation of par- 
asitic effects of hard-nonlinearities, based on static and dynamic compensators that use neural networks. It is known that various control techniques with tuning algoritms are developed for common actuator nonlinearities that guarantee small tracking error and bounded internal states, too. On the other hand, we present here the common approach based on extraction of an unmeasurable internal signal, acting between linear and nonlinear blocks of the Wiener system, avoiding design of compensators. We propose here to extract an internal signal using the trivial rearrangement of observations. Two parametrical models of the linear block and the iterative technique, based on ordinary LS, are used. The simulation results, presented in Figs. 5-21, imply that the iterative technique, based on the input-output data rearrangement and on the FIR model (5), where such reordered observations have been used to fill the respective matrix (8) and vectors (10)-(12) differently for each hard-nonlinearity, rejecting cut-off observations, could be used for the iterative estimation of unknown parameters of Wiener systems. Later on, the internal signal is extracted by formula (32). It appears that with an increase of the amount of observations to be processed, the accuracy of estimates also slightly grows because of the increased number of incorrect observations. The additive process and measurement noises in observations to be processed strongly influence the quality of parametric identification of the Wiener system with a discontinuous nonlinearity.

\section{References}

Armstrong-Hélouvry, B., Dupont, P., Canudas de Wit, C. (1994). A survey of models, analysis tools and compensation methods for the control of machines with friction. Automatica, 30(7), 1083-1138.

Åström, K.J., Wittenmark, B. (1996). Computer-Controlled Systems: Theory and Design, 3rd edn. Prentice Hall, New York.

Bai, E.W. (2002). Identification of linear systems with hard input nonlinearities of known structure. Automatica, $38,853-860$.

Canudas de Wit, C., Olsson, H., Åström, K.J., Lischinsky, P. (1995). A new model for control of systems with friction. Transactions on Automatic Control, 40(3), 419-425.

Desoer, C.A., Shahruz, S.M. (1986). Stability of dithered nonlinear systems with backlash or hysteresis. International Journal of Control, 43(4), 1045-1060.

Hagenblad, A. (1998). Identification of Wiener models. Technical report LiTH-ISY-R-2031, Department of Electrical Engineering, Linköping University, SE-581 83 Linköping, Sweden.

Ljung, L. (1999). System Identification. Theory for the User, 2nd edn. Prentice-Hall, New York.

Malinvaud, E. (1969). Statistical Methods of Econometrics. North-Holland, Amsterdam.

Pupeikis, R. (2003). Identification of piecewise affine Wiener systems using data partition. Technical report LiTH-ISY-R-2523, Department of Electrical Engineering, Linköping University, SE-581 83 Linköping, Sweden.

Pupeikis, R. (2005). On the identification of Wiener systems having saturation-like functions with positive slopes. Informatica, 16(1), 131-144.

Pupeikis, R. (2005). On the identification of Hammerstein-Wiener systems. Lietuvos matematikos rinkinys, 45(spec. nr.), 509-514.

Pupeikis, R. (2011). On recursive parametric identification of Wiener systems. Information Technology and Control, 40(1), 21-28

Pupeikis, R., Navakauskas, D., Ljung, L. (2003). Identification of Wiener systems with hard and discontinuous nonlinearities. Technical report LiTH-ISY-R-2501, Department of Electrical Engineering, Linköping University, SE-581 83 Linköping, Sweden.

Selmic, R.R., Lewis, F.L. (2000). Deadzone compensation in motion control systems using neural networks. IEEE Transactions on Automatic Control, 45(4), 602-613. 
Selmic, R.R., Lewis, F.L. (2001). Neural net backlash compensation with Hebbian tuning using dynamic inversion. Automatica, 37, 1269-1277.

Selmic, R.R., Phoha, V.V., Lewis, F.L. Intelligent Compensation of Actuator Nonlinearities. http://www2.latech.edu/ phoha/PDF.

Schoukens, J., Pintelon, R., Dobrowiecki, T., Rolain, Y. (2005). Identification of linear systems with nonlinear distortions. Automatica, 41, 491-504.

Widanage, J., Godfrey, K.R., Tan, A.H. (2004). System identification in the presence of a saturation nonlinearity. Journal of Electrical Engineering, 55(1-2), 46-49.

Vörös̈, J. (2001). Parameter identification of Wiener systems with discontinuous nonlinearities. Systems \& Control Letters, 44, 363-372.

Vörös, J. (2002). Modeling and parameter identification of systems with multisegment piecewise-linear characteristics. IEEE Trans. Automatic Control, 47(1), 184-188.

Vörös, J. (2010). Recursive identification of systems with noninvertible output nonlinearities. Informatica, 21(1), 139-148.

Vörös, J. (2010). Block-oriented nonlinear system identification. Lecture Notes in Control and Information Sciences, 404/2010, 35-51.

Vörös, J. (2011). Identification of nonlinear cascade systems with time-varying backlash. Journal of Electrical Engineering, 62(2), 87-92.

Wigren, T. (1993). Recursive prediction error identification using the nonlinear Wiener model. Automatica, 29(4), 1011-1025.

\section{Apie sumanuji vidinio signalo išgavimą Vinerio sistemoje, susidedančioje iš tiesinio bloko ir po jo sekančio kieto netiesiškumo}

\section{Kazys KAZLAUSKAS, Rimantas PUPEIKIS}

Straipsnyje nagrinejjamas Vinerio sistemu su dinamine tiesine dalimi ir po jos sekančio kieto netiesiškumo netiesinejje dalyje nematuojamo vidinio signalo išgavimo uždavinys. Parodyta, kad šis uždavinys, pertvarkius ièjimo bei užtriukšminto išèjimo signalų stebejjimus ir juos atskyrus, suvedamas ị tiesinị parametrinio vertinimo uždavinị. Pasiūlyta nuosekli Vinerio sistemos vidinio signalo, esančio tarp netiesinès ir tiesinès dalių atkūrimo procedūra ịvairiems kietiems netiesiškumams triukšmų aplinkoje. Gautos šio signalo einamosios reikšmès gali būti taikomos rekurentiniams abiejų dalių koeficientų iverčiams gauti. Pateikti Vinerio sistemų su penkiais kietais netiesiškumais modeliavimo, ju parametrinio identifikavimo bei vidinio nematuojamo signalo išgavimo rezultatai. 